\title{
openSAP: Why Are Enterprise MOOCs Working?
}

\author{
https://doi.org/10.3991/ijac.v12i3.11262 \\ Jan Renz $\left.{ }^{(}\right)$, Christoph Meinel \\ Hasso Plattner Institute, Potsdam, Germany \\ jan.renz@hpi.de \\ Clemens Link \\ SAP, Walldorf, Germany
}

\begin{abstract}
SAP is one of the first and leading companies offering free online courses. After more than five years of offering openSAP.com, this paper takes a look at insight gained during this time and aims to identify success factors for enterprise MOOC providers.
\end{abstract}

Keywords-MOOCs, lifelong learning, Enterprise MOOCs, workplace learning

\section{Introduction}

The rapid growth and changes in technologies demand a rapid transfer of knowledge in many areas. Not only in the education sector, but also in the economy, opportunities for a fast flow of information are very important. SAP meets these growing requirements with its Enterprise MOOC platform openSAP and thus meets the growing need to flexibly train employees, but also consultants, users and deciders. The aim is to convey knowledge about new technologies quickly and directly to the target group.

\section{Easy Access for an Open Community}

In April 2013, SAP launched its first courses on the openSAP MOOC platform and was the first company to invest in MOOCs and its own platform. This made it possible to offer free courses for an open user community. Originally it was planned to run the platform only as a short-term project to enable developers to get upskilled on SAP HANA development. The goal was to reach as many people as possible.

The success confirms this effort. While around 55,000 learners (40 thousand while runtime) had registered for the first course, in May 2019 more than 820,000 learners from all were included in the user group of the offer. With the increasing interest, the range of courses has been steadily expanded and today more than 180 courses are offered on openSAP. 
Table 1. Enrollments and learners

\begin{tabular}{|c|c|c|}
\hline Year & New course enrollments & New unique learners \\
\hline 2013 & 17,000 & 79,846 \\
\hline 2014 & 332,450 & 90,787 \\
\hline 2015 & 500,607 & 123,532 \\
\hline 2016 & 651,304 & 156,769 \\
\hline 2017 & 654,380 & 144,808 \\
\hline 2018 & 794,552 & 153,019 \\
\hline
\end{tabular}

The target group of the courses offered on openSAP are developers, users, other stakeholders and last but not least SAP employees. However, the courses are open to all interested parties. Learners only need Internet access and a user account on openSAP to participate in the courses, which can be easily created as long as a user has a valid and active email account. Learners with an existing SAP account can also log in to openSAP via this account, which further minimizes the access hurdles.

The open nature of this learning format makes it possible to make knowledge quickly and easily available to all target groups, while the MOOCs approach gives the participants a great deal of freedom and flexibility. They can divide their learning times as freely as possible and they are not constrained to a fixed place of learning. This makes it possible to reach learners all over the world - across different time zones and independent of stationary training locations.

\section{Audience and Usage}

Within the first five years, more than three million enrollments can be counted for the courses, which means an average of more than four courses per user. The average amount of enrollments per course (measured at the course end) is 9,400. Of all learners registered for the course, about 50 percent participate actively in the course and 25 percent complete the course successfully (Record of Achievement). These evaluations are based on the numbers of registered participants enrolled to the course at the halfway point, since it is to be assumed that the time for participants starting later is no longer sufficient to complete the earlier weeks and to collect enough points for the successful completion.

On openSAP different course types are offered. The standard courses have a duration of six weeks with weekly new published contents. In addition, there are nutshell courses that give an overview of a topic with all content available from the start of the course and the content in total takes about four hours to complete over a four-week period.

Further investigation shows some differences: learners who enroll during a course are more active (over 40 percent of 75 percent course active learners) and most likely to earn a Record of Achievement (RoA) or Confirmation of Participation (CoP) during the course run time when compared to learners who enroll before a course starts. (see Fig. 1 and Fig. 2). 


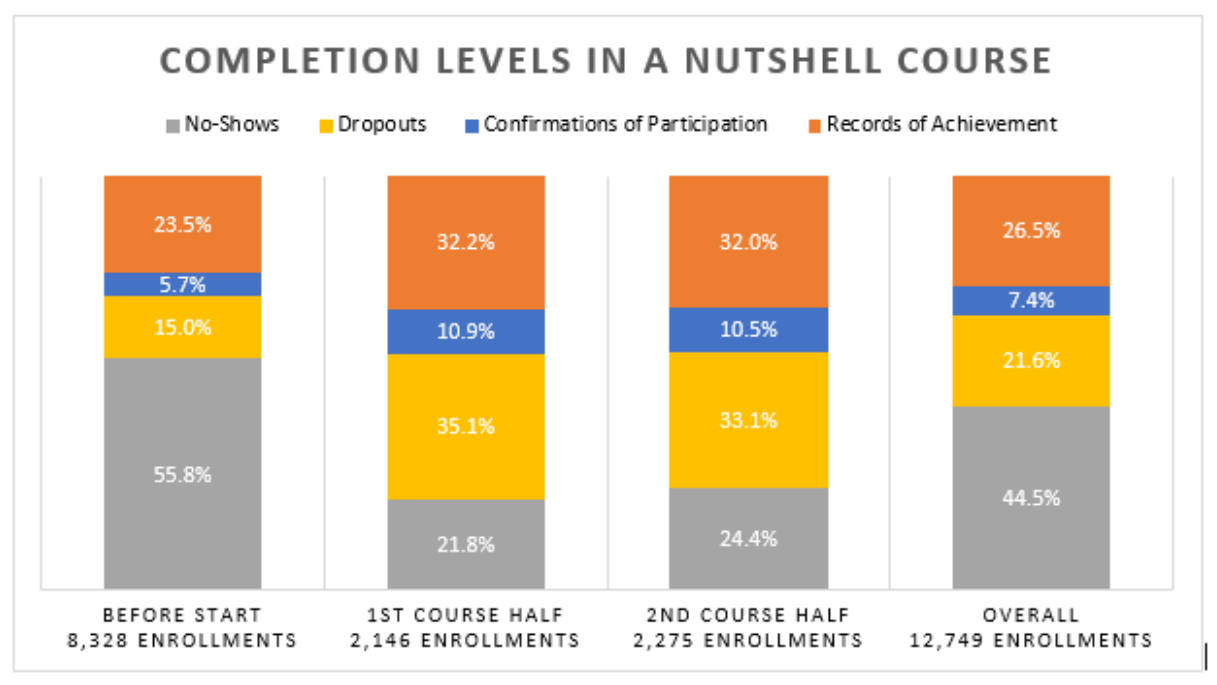

Fig. 1. Example for a nutshell course

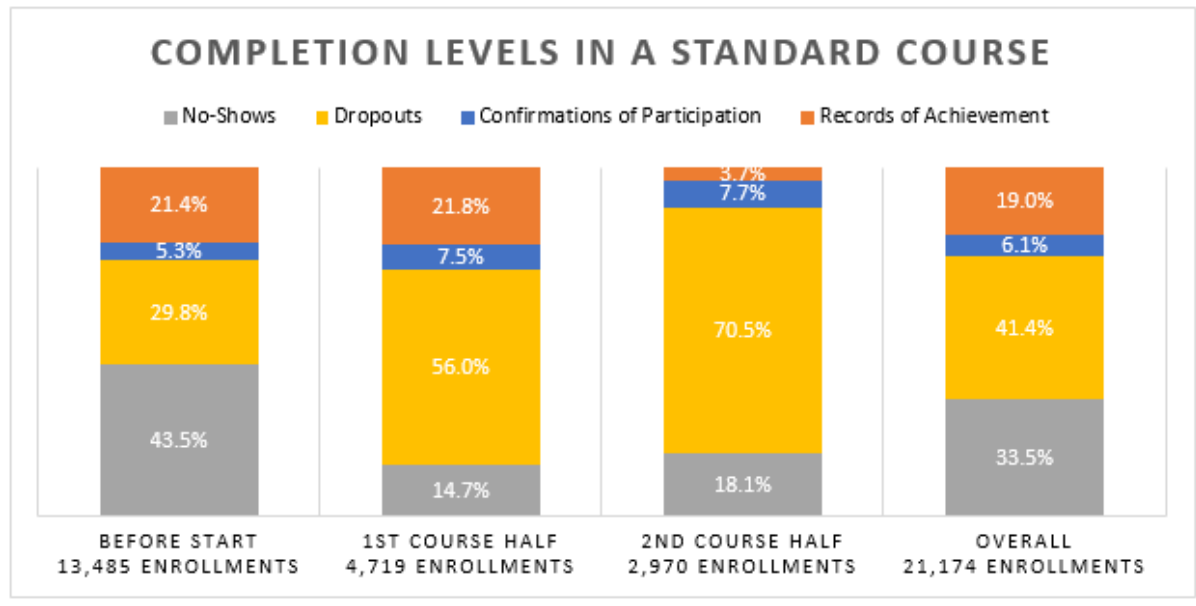

Fig. 2. Example for a standard course

The monthly based tracking the number of learners who actively take part in courses shows some peaks. While the average number of active users in many months is 50 thousand, there are some months in which about 70 thousand active users were to be seen, for example April and May 2019 with popular topics featured on openSAP including SAPUI5 and SAP Cloud Platform. (see Fig. 3) 


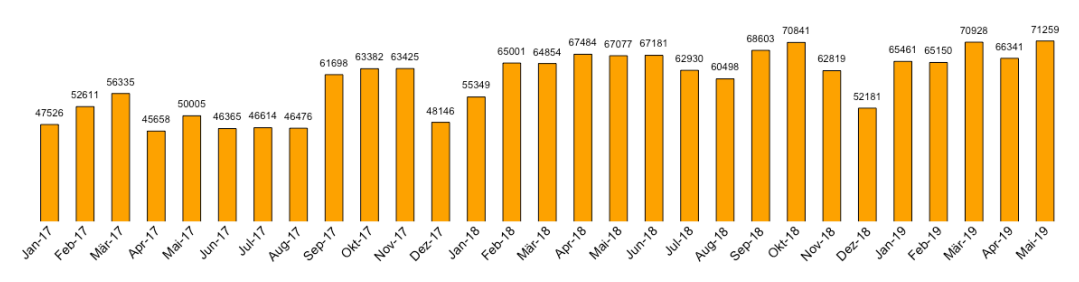

Fig. 3. Active learners at openSAP

Based on the learners' age distribution (see Fig. 4) openSAP clearly belongs to lifelong learning. Based on surveys that are conducted at the end of each course and have been answered more than 150,000 times, it can be stated that: 98 percent of the survey participants are (very) satisfied with the openSAP learning experience. Ninety-five percent think the skills taught in the course will help them in their (future) work while 99 percent plan to take additional openSAP courses in the future. The Net Promoter Score (average) is above 50, which can be considered a very good value.

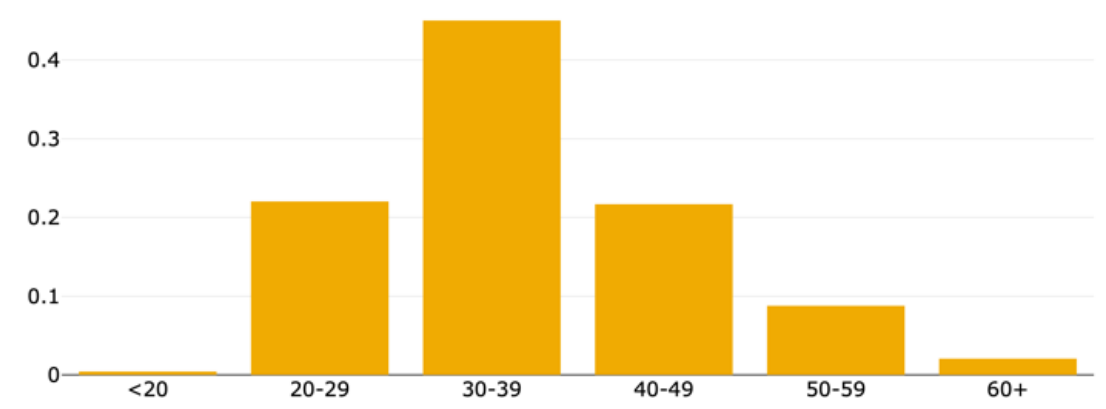

Fig. 4. Age distribution (based on non-mandatory profile data)

\section{Course Structure and Format}

openSAP follows a traditional xMOOC format. Typical for this format is the structured preparation and structuring of the material into individual sections (usually one per week) which contain the individual learning items. The typical course duration is four to six weeks. Most courses offer a linear learning path; however, some elements may be optional. In a few courses, individual learning paths with alternative sections are offered. New content is activated at the beginning of each course week. If several courses run in parallel on the platform, the activation and the dating of the weekly homework takes place distributed on the individual weekdays. Whereas this was originally introduced in order to avoid peak loads (high access rates can be observed especially after the activation of new content and before the expiry of deadlines), today this 
mainly serves the purpose to accommodate learners who attend several courses at the same time.

Each week there is a repetitive sequence of video-based knowledge transfer and knowledge verification via automated self-tests. At the end of a week, usually a homework task with points follows. This structure is supplemented by the provision of collaboration rooms and a forum integrated into the learning platform.

There is a final examination at the end of the course, which usually grants 50 percent of the total points available, while the remaining points can be achieved through the weekly homework. In general, the "graded" tasks only allow one attempt, which is limited to 60 minutes.

In addition to surveys based on the quiz module, it is also possible to leave textual feedback in an "I like, I wish" style based on the design thinking methodology.

Some courses offer peer assessment or team peer assessment tasks. These allow for more flexible tasks that go well beyond simple quizzes.

\section{$5 \quad$ Success Factors}

In this chapter we identify different success factors that we assume are responsible for the sustainable success of openSAP and Enterprise MOOCs in general.

\subsection{Provide enough team resources and an interdisciplinary team}

The courses are developed by a team of 20 people, who bring experts from the respective departments into the team to provide technical support and domain knowledge for specific courses. Seven teams are involved in course development and execution at the operational level. The Helpdesk team answers questions from users. These questions can be sent via a form contained in the portal. At the same time, data about the browser is used and the course context is attached to the helpdesk ticket. So that helpdesk tickets can be processed promptly, the employees are distributed in several time zones.

The communications department takes care of design and content. In addition to the brand-specific design of the platform, the design of course certificates and open badges, this also includes course communication in the form of course-specific and global news, which are available within the platform and the apps, but are also sent to the learners by e-mail. It is also responsible for releasing the slide decks used within the courses. In addition to compliance with the Corporate Style Guides, legal clarification of copyrights is also taken into account during this process. The Instructional Designers provide cross-course advice on media and, in particular, self-test creation and continuously monitor solution rates. Each course is supervised by a dedicated project manager over its entire duration, i.e. from conception, creation, active duration and cool-down. They are accompanied by content experts.

The operations team is responsible for uploading the content to the platform, including final quality control. This team is also responsible for sending course- and platformwide messages to all users. Even if the platform allows these activities to be carried out 
by the respective course supervisors, such centralization allows communication measures and deadlines to be coordinated. In particular, users who participate in several courses at the same time can be taken into consideration.

\subsection{Ownership of production workflows}

In traditional learning environments, workflows of production were often related to the development and updating of content involving external agencies. MOOCs and their primary video-based content delivery channel allow easy production workflows. Content experts can produce content themselves using screen recording software. Delivering the content in small content items also allows easy updating of course content, by extending or updating the courses.

\subsection{Offer relevant topics}

Courses are tagged with high level topics, where one course can be tagged with more than one topic.

A dedicated program manager has the task to combine the demands of the learner's community with the teams from within SAP that want to offer courses. Surveys and the "I like, I wish" forum help to get qualitative and quantitative feedback on desired content. As shown in Table 1, there is a good mix from different topics. The task of the program manager is also to orchestrate and distribute the courses over the year (courses are offered throughout the year, excluding breaks in summer and winter holidays).

Table 2. Overview of courses by topic

\begin{tabular}{|l|c|}
\hline \multicolumn{1}{|c|}{ Topic } & No of courses \\
\hline User Experience & 34 \\
\hline SAP Cloud Platform & 30 \\
\hline Cloud Solutions & 43 \\
\hline Social Responsibility & 17 \\
\hline Analytics & 32 \\
\hline Enterprise Management & 31 \\
\hline SAP HANA & 25 \\
\hline Digital Transformation & 34 \\
\hline Human Resources & 11 \\
\hline SAP S/4HANA & 25 \\
\hline Continuous Innovation & 20 \\
\hline Industries & 16 \\
\hline
\end{tabular}

\subsection{Course promotion}

Courses are published on the platform about six weeks before the start of the course and advertised in regular platform-wide messages, which are also sent to all users by email. From this point on it is possible to register for the courses.

While course announcements reach more than one-third of the enrolled users (users that either read the mail or open the announcement in the app or on the web page), global announcements are opened up by an average of 20 percent of all users. Excluding 
users that deactivated mail notifications or have been marked inactive due to non-working email accounts, this means around 120,000 users are reading global news on average. Additional marketing is conducted through social media sites, SAPs website and the company's social communities. No paid promotion is used to gain new users.

\subsection{The xMOOC course format}

The courses always follow a fixed schedule. The content is outlined on an introductory description page, the lecturers are introduced, and there is also information on the target group and any necessary access requirements. The courses are structured on a weekly basis. Each block consists of instructional videos followed by self-tests and a final, scored weekly test. In addition to the tests, access to systems is also offered where possible so that the acquired knowledge can be evaluated in a practical way. At the end of the course the participants can take a final test and receive a certificate after passing. A forum is available to the user during the course. Here the learners can communicate with each other and with the support team. This xMOOC typical format is well established in lifelong learning on many platforms like edX and openHPI, still SAP was one of the pioneers in adapting and applying this to enterprise learning.

The idea to use mobile apps to access the course content through mobile apps was sketched in the early beginnings of the project [5]. While most usage of the platform is conducted through desktop devices and a web browser (desktop web), there is also a significant usage through a web browser on a mobile device and the native apps, which are offered for iOS and Android. Most of the users that are using the apps or the mobile web page also use the web version on a desktop class device.

\subsection{Apps and mobile usage}

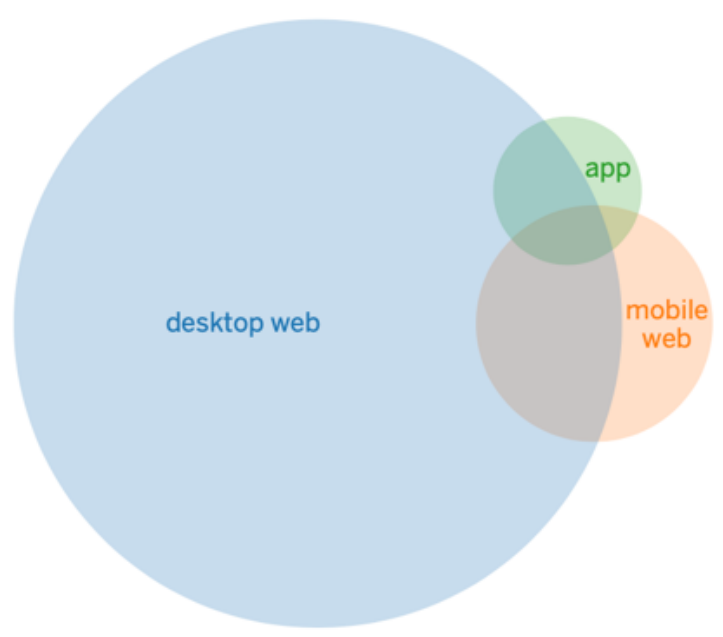

Fig. 5. Desktop, mobile and app usage 
For the app users, two-thirds of the time spent within the apps is used watching videos (65 percent on iOS and 67.5 percent on Android)

\subsection{Allow easy coexistence with daily work}

As shown in the activity heat map, openSAP is mainly used during working hours. Also, there is more usage in the early evening hours in the beginning of the week. This could be related to more motivation after a recreational weekend, but also to the fact that new content is published at the beginning of the week and learners like to get things done. In general, the main usage seems to be after other office tasks have been finished, as it can be seen by the relative low usage on Monday morning. While there is more activity on workdays, the sessions duration is higher on Sundays, indicating that learners that learn in their private surroundings tend to learn in longer sessions and learning in the workplace is happening in smaller chunks.

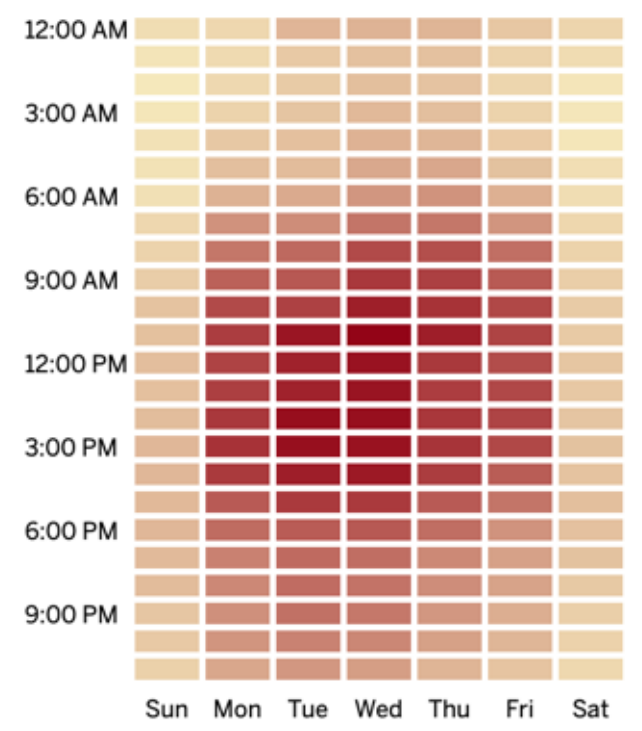

Fig. 6. Learners activity heat map by local time and date

Concepts like the usage of personal learning objectives that are described in [4] and have recently been tested on openSAP allow the learners to concentrate on those parts of the course that they are interested in, while still being able to track the progress of the learning objectives in a dashboard.

\subsection{Regular course communication}

Unlike webinars or onsite seminars, MOOCs require learners to proactively access the content themselves. This requires them to remember they are part of a running course and go to the website or open the mobile app. To minimize this hurdle, regular 
communication is key to success. This communication takes place in the course announcements that are send out by mail and are available online but also through automated emails.

The enrollment mail is sent out automatically after the user's enrollment and contains basic information about the course like deadlines and tips on how to learn. A few days before the course starts a reminder mail is sent out. During the course an announcement mail is sent out each week when new content is published or deadlines approach. High opening rates and activity after the sending prove the effectiveness of this communication channel.

Users that engage in the course forums also receive forum notification mails when there is activity in a thread, they participated in. They can also opt-in to a daily forum digest mail. It is also planned to send out this notification via Push Notification to the mobile apps.

Automated reminder mails have been tested in an $\mathrm{AB}$ experiment, described in [3] and proved that automated mails also increased the course activity of those users that received those mails significantly. It also showed that the course videos, but not the community-contributed content, in the forums is the trigger to re-enter the course.

\subsection{Interactive and peer assessments}

Multiple-choice tests are a good fit for self-tests and graded assessments, but these assessment formats have a limited flexibility. Enabling assessments that will lead to a more in-depth reflection based on Bloom's revised taxonomy [6], while still allowing gradeability without requiring too much workload from the teaching team.

For a few coding introduction courses an open-source programming platform was used [7]. For other courses, a (team) peer assessment workflow was added to the platform. This allowed assessments like "write a concept for an app" or "write an app", that led to over 1000 apps submitted in one course.

\subsection{Providing benefits for the learner}

This format offers several advantages for learners. Access to the resources is free of charge, there is no maximum number of participants and only an email address is required for registration. Participants also enjoy great flexibility in the choice of learning location and time, and access is possible on all devices. At the same time, the smallscale content allows the learning process to be spread over several learning sessions. This allows learners to work at their own working speed and, if necessary, repeat individual parts. Motivational learning is to be achieved through certificates, high-quality content and gamification.

\subsection{Providing benefits for the provider}

Early after the start of the pilot phase, it was foreseeable that the openSAP platform would become a long-term project due to the positive feedback, the significantly higher 
than expected number of users and the internal demand from potential course providers. This was accomplished by a corresponding organizational structure and continuity.

One of the main advantages for the provider is that knowledge can be disseminated much faster and more efficiently than by conventional means. New courses can be produced quickly and efficiently. Costs for classroom training can be avoided and the geographical range can be extended. In addition, the forum offers a valuable channel for collecting customer feedback within the courses. This not only involves feedback on the courses themselves, but also on the content of the company's products and services. At the end of a course, learners are asked for feedback in the form of a short survey. According to this survey, 99 percent of the participants want to attend further courses. The learning experience is assessed as positive by 98 percent, with 95 percent of learners stating that they find the offer relevant for their professional development. These figures also confirm the success of the model for SAP.

\subsection{Co-Development and research}

The cooperation with the Hasso Plattner Institute has also proven to be a goal-oriented approach for openSAP. As a result of the research cooperation, joint solution concepts such as the Team Builder [1], the (Team) Peer Assessment and offline capable apps have been developed. The focus here is on approaches that combine the requirements from the company's and the learner's point of view.

The deep integration into the existing company IT, such as the possible single signon of the users, allows an easy use and also the provision of internal course offers for certain target groups. Through scientific monitoring and evaluation, these measures can not only be evaluated and improved in themselves, they can also be strategically further developed. Learning analytics and reports, enabled by [2] are an important building block of this. Since the high level of activity on the platform allows quantitative testing of theoretical concepts, openSAP is also of interest from the Institute's point of view (HPI) and allows AB-Testing [3]. While this close and project-centric view makes sense in the early years of the project, in later days a more product-based approach (while still being agile and user-centric) should be followed to increase sustainability.

\section{Conclusion}

After more than five years of running openSAP, a list of success factors for Enterprise MOOCs has been identified. While these factors are important, they will only work if the fundamentals are set: interesting content that is relevant for the learners, presented by authentic course hosts, and provided on a platform that enables a good learner experience. Providing a platform like openSAP should be understood as a process that is focused on steady optimization. This includes course formats and concepts, but also the learning experience based on the platform. The large number of learners enables a set of quantitative methods like learning analytics, AB-testing, automated quality control, polls, and surveys that should be used to reach a win-win situation for enterprises and learners. 


\section{$7 \quad$ References}

[1] Staubitz, Thomas, and Christoph Meinel. "Collaboration and Teamwork on a MOOC Platform: A Toolset." Proceedings of the Fourth (2017) ACM Conference on Learning@ Scale. ACM, 2017. https://doi.org/10.1145/3051457.3053975

[2] Renz, Jan, et al. "Enabling schema agnostic learning analytics in a service-oriented MOOC platform." Proceedings of the Third (2016) ACM Conference on Learning@ Scale. ACM, 2016. https://doi.org/10.1145/2876034.2893389

[3] Renz, Jan, et al. "Using A/B testing in MOOC environments." Proceedings of the Sixth International Conference on Learning Analytics \& Knowledge. ACM, 2016. https://doi.org/10.1145/2883851.2883876

[4] Rohloff, Tobias, and Christoph Meinel. "Towards Personalized Learning Objectives in MOOCs." European Conference on Technology Enhanced Learning. Springer, Cham, 2018. https://doi.org/10.1007/978-3-319-98572-5_16

[5] Renz, Jan, Thomas Staubitz, and Christoph Meinel. MOOC to Go. International Association for the Development of the Information Society, 2014.

[6] Anderson, Lorin W., et al. "A taxonomy for learning, teaching, and assessing: A revision of Bloom's taxonomy of educational objectives, abridged edition." White Plains, NY: Longman (2001).

[7] Staubitz, Thomas, et al. "CodeOcean-A versatile platform for practical programming exercises in online environments." 2016 IEEE Global Engineering Education Conference (EDUCON). IEEE, 2016. https://doi.org/10.1109/EDUCON.2016.7474573

\section{Authors}

Jan Renz is with the Hasso Plattner Institute (e-mail: jan.renz@hpi.de) and part of the Knowledge and Learning Engineering team.

Christoph Meinel is the CEO of the Hasso Plattner Institute (e-mail: christoph.meinel@hpi.de).

Clemens Link is the founder of OpenSAP and working at SAP in Walldorf, Germany. (e-mail: clemens.link@sap.com).

This article is a revised version of a paper presented at the International Conference on E-Learning in the Workplace 2019 (ICELW 2019), held in June 2019, at Columbia University in New York, NY, USA. Article submitted 2019-07-12. Resubmitted 2019-08-13. Final acceptance 2019-08-22. Final version published as submitted by the authors. 